\title{
A Novel Frequency-Offset Monitoring Technique for Direct-Detection DPSK Systems
}

\author{
H. C. Ji, Paul K. J. Park, Member, IEEE, Hoon Kim, Member, IEEE, J. H. Lee, and Y. C. Chung, Fellow, IEEE
}

\begin{abstract}
We propose and demonstrate a novel technique to monitor the frequency offset between the optical source and delay interferometer (DI) for direct-detection differential phase-shift-keying (DPSK) systems. In this scheme, a phase-modulated tone is applied to DPSK signals at the transmitter and then detected after being converted into an amplitude-modulated tone at the DI to be used for the monitoring signal. Our experimental demonstration shows that the monitoring range and sensitivity of the proposed scheme are measured to be $\pm 2 \mathrm{GHz}$ and $\sim 10 \mathrm{MHz}$, respectively, which we believe are good enough to be used either to generate alarm signals for the frequency offset monitoring or to control the feedback loop of the DI.
\end{abstract}

Index Terms-Delay interferometer (DI), differential phase-shift keying (DPSK), frequency offset, monitoring, phase modulated (PM) tone, polarization-dependent wavelength shift.

\section{INTRODUCTION}

$\mathbf{R}$ ECENTLY, the differential phase-shift-keying (DPSK) modulation format has attracted a great deal of attention for long-haul high-capacity transmission systems. This is mainly because this modulation format offers $\sim 3-\mathrm{dB}$ improvement in receiver sensitivity over conventional ON-OFF keying when a balanced receiver is used for an optically preamplified receiver [1]. In addition, the pattern-independent optical intensity of the DPSK signal could mitigate some fiber nonlinearities such as cross-phase modulation and self-phase modulation [2], [3]. A direct-detection DPSK system typically utilizes a delay interferometer (DI) for signal demodulation at the receiver. For the proper operation of this system, it is essential to align the wavelength of the DI with that of the optical source since even a slight misalignment results in large performance degradation [4], [5]. For example, it has been reported that a frequency offset of 300-400 MHz between the DI and optical source gives rise to $1-\mathrm{dB}$ sensitivity penalty in $10-\mathrm{Gb} / \mathrm{s}$ DPSK systems. In real transmission systems, the frequency offset can be induced by 1) wavelength drift of the optical source, 2) polarization-dependent wavelength shift $(\mathrm{PD} \lambda)$ of the DI in the presence of polarization rotation of the signal [5], and 3) thermal drift or

Manuscript received December 5, 2005; revised January 25, 2006.

H. C. Ji was with the Department of Electrical Engineering and Computer Science, Korea Advanced Institute of Science and Technology, Daejeon 305-701, Korea, and is now with the Photonic Network Laboratory, Osaka University, Osaka 565-0871, Japan (e-mail: hcji5002@ gmail.com).

P. K. J. Park is with the Digital Solution Center, Samsung Electronics, Seoul 135-524, Korea.

H. Kim is with the Telecommunications R\&D Center, Samsung Electronics, Suwon 442-600, Korea.

J. H. Lee and Y. C. Chung are with the Department of Electrical Engineering and Computer Science, Korea Advanced Institute of Science and Technology, Daejeon 305-701, Korea.

Digital Object Identifier 10.1109/LPT.2006.872325

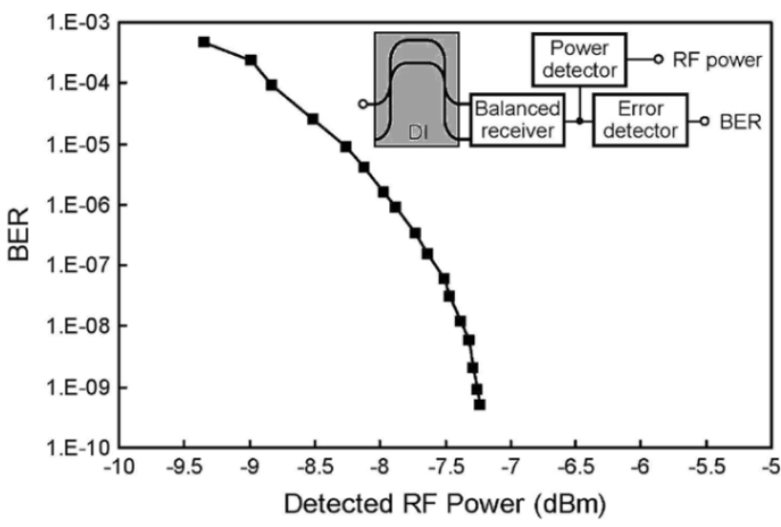

Fig. 1. Measured BER versus the RF power of the received DPSK signals when the performance of the DPSK signal is degraded by the frequency offset. The inset shows the frequency offset monitoring scheme based on RF power detection.

aging of the DI itself. Therefore, it is necessary to monitor the frequency offset between the DI and optical source during the operation of the systems.

Previously, the frequency offset was monitored by measuring the dc photocurrent of the balanced receiver or radio-frequency (RF) power of the received DPSK signals [6], [7]. In [6], it has been proposed to monitor the frequency offset by measuring the carrier leak through at the balanced receiver, which is generated by utilizing slightly less than $V_{\pi}$ phase modulation (PM) at the transmitter. Therefore, this technique cannot be applied to the DPSK transmitter using a Mach-Zehnder (MZ) modulator since nearly perfect $\pi$ phase shift is always achieved with the MZ modulator. Another way to monitor the frequency offset is to measure the total RF power of the received DPSK signals [7]. Since the frequency offset causes eye closure, the RF power reduction could be used as an indication of the frequency offset. Fig. 1 shows the measured bit-error ratio (BER) as a function of the received RF power when we intentionally increase the frequency offset between the DI and optical source. The frequency offset monitoring scheme based on RF power detection is also depicted in the inset. As shown in this figure, the major drawback of this scheme is that the monitoring sensitivity is too poor to be used for the monitoring signal especially when the BER is low. For example, only a 0.1-dB RF power drop is observed for a BER change from $10^{-9}$ to $10^{-8}$.

In this letter, we propose and demonstrate a way to monitor this frequency offset with a high sensitivity. In this scheme, we apply a PM tone to the DPSK signal at the transmitter and then measure the tone magnitude after PM-to-amplitude-modulation (AM) conversion at the DI. Therefore, this scheme can be used for a DPSK transmitter based on MZ modulators as well as phase modulators. Our experimental results show that 


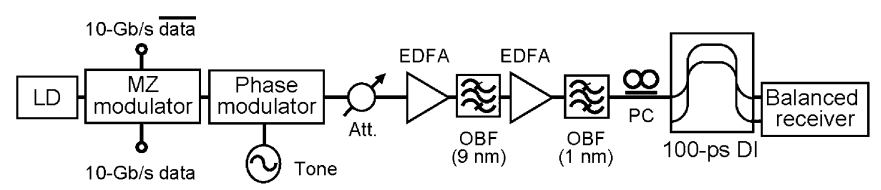

Fig. 2. Experimental setup.

the monitoring range and sensitivity of the proposed technique are $\pm 2 \mathrm{GHz}$ and $\sim 10 \mathrm{MHz}$, respectively.

\section{THEORY}

A DPSK receiver is composed of a 1-bit DI and a balanced receiver. When the DPSK signal is dithered with a PM tone at angular frequency $\Omega$ for the monitoring, the normalized signal incident on the DI can be described as

$$
s(t)=e^{j \omega t} e^{j \Delta \omega t} e^{\Phi(t)} e^{j A \cos (\Omega t+\phi)}
$$

where $\omega$ is the angular frequency of the optical signal, $\Delta \omega$ is the angular frequency offset between the DI and optical source, $\Phi(t)$ is the phase of the DPSK signal carrying data information, $A$ is the amplitude of the PM tone, and $\phi$ is the phase of the PM tone. In the DI, the PM described in (1) is converted into AM. Then, the outputs at the constructive and destructive ports of the DI can be expressed as

$$
\begin{aligned}
& p(t)=s(t)+e^{j \pi} s(t-T) \\
& q(t)=e^{j \frac{\pi}{2}} s(t)+e^{j \frac{\pi}{2}} s(t-T)
\end{aligned}
$$

respectively. If we assume that the phase deviation caused by dithering is much smaller than the phase difference of DPSK signal (i.e., small PM index), the output of the balanced receiver can be expressed as

$$
\begin{aligned}
r(t)= & |p(t)|^{2}-|q(t)|^{2} \\
= & \left(4-\xi^{2}\right) \cos \kappa(t)-4 \xi \sin \kappa(t) \cos (\Omega t+\varphi) \\
& -\xi^{2} \cos \kappa(t) \cos (2 \Omega t+2 \varphi)
\end{aligned}
$$

where $\xi=A \sqrt{2(1-\cos \Omega T)}, \varphi=\phi-$ $\tan ^{-1}(\sin \Omega T /(\cos \Omega T-1))$, and $\kappa(t)=\pi+\Phi(t-$ $T)-\Phi(t)-\omega T-\Delta \omega T$. If there is no frequency offset (i.e., $\Delta \omega=0$ ) in a DPSK system, the value of $\kappa(t)$ would be either zero or $\pi$, implying that there is no AM tone at $\Omega$ (i.e., fundamental component), whereas the AM-tone amplitude at $2 \Omega$ (i.e., second-harmonic component) becomes maximum. However, as the frequency offset is raised, the fundamental component increases to $|4 \xi \sin (\Delta \omega T)|$ but the second-harmonic component is reduced to $\left|\xi^{2} \cos (\Delta \omega T)\right|$. For small frequency offset, the amplitude of the fundamental component can be approximated to $4 \xi \Delta \omega T$. Owing to this linear relation between the frequency offset and the amplitude of AM tone, the PM-to-AM converted fundamental tone can be used to monitor the frequency offset of the DPSK systems.

\section{RESULTS AND DISCUSSION}

Fig. 2 shows the experimental setup used to demonstrate the proposed frequency-offset monitoring technique in a DPSK system. The output of a distributed feedback (DFB) laser

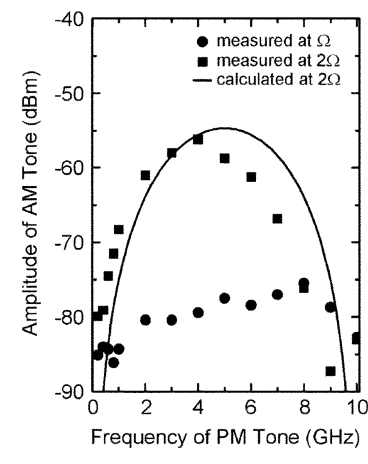

(a)

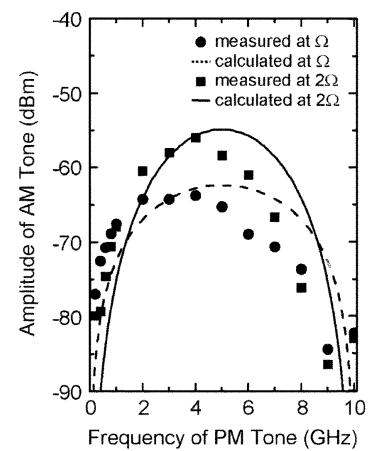

(b)
Fig. 3. RF power of the PM-to-AM converted tones as a function of the PM-tone frequency (a) without and (b) with 300-MHz frequency offset.

operating at $1558.5 \mathrm{~nm}$ was modulated with a $10-\mathrm{Gb} / \mathrm{s}$ nonreturn-to-zero (NRZ) signal (pattern length: $2^{31}-1$ ) using a dual-drive MZ modulator to generate NRZ-DPSK signals. We then dithered the phase of the DPSK signals by using a $\mathrm{LiNbO}_{3}$ phase modulator. The DPSK receiver was composed of a two-stage erbium-doped fiber amplifier, optical bandpass filters, a 100-ps DI, and a balanced receiver. The PD $\lambda$ of the DI was $300 \mathrm{MHz}$. The RF power of the AM tone, generated by the PM-to-AM conversion of the DI, was measured by using an RF spectrum analyzer. In a real DPSK system, a narrow bandpass filter followed by an RF power detector can take the place of the RF spectrum analyzer.

We first optimized the frequency and modulation index of the PM tone. The PM tone should be large enough to discern the monitoring signals from noise-like data signals but also small enough not to induce significant sensitivity degradation for DPSK signals. Fig. 3 shows the measured AM-tone power together with the calculated curves from (3) as a function of PM-tone frequency. In the absence of the frequency offset as depicted in Fig. 3(a), the fundamental component is observed to be as small as the instrument noise and the second-harmonic component shows the maximum intensity near half the data rate. The discrepancy between the measurement and calculation for the second-harmonic component at $>5 \mathrm{GHz}$ should be attributed to the receiver bandwidth of $10 \mathrm{GHz}$ : when the $\mathrm{PM}$ tone is higher than $5 \mathrm{GHz}$, the second-harmonic component would be higher than $10 \mathrm{GHz}$ and would fall outside the receiver bandwidth. When there exists a frequency offset of $300 \mathrm{MHz}$, as shown in Fig. 3(b), both the fundamental and the second-harmonic components exhibit the maximum intensity at half the data rate. Therefore, to maximize the readability of the AM fundamental component at the receiver, the tone frequency should be chosen around half the data rate. At these tone frequencies, however, the sensitivity degradation of the DPSK signal is maximized as well. Fig. 4(a) shows the sensitivity penalty versus PM-tone frequency. In this measurement, we set the optical signal-to-noise ratio (OSNR) to be $30 \mathrm{~dB}$. However, it should be noted that amplified spontaneous emission (ASE) noise cannot degrade the performance of the proposed technique even if the OSNR is much lower than $30 \mathrm{~dB}$. This is because that in typical optical transmission systems, the tone SNR would be determined by noise-like random data rather than ASE noise or receiver noise. In addition, the tone SNR could be enhanced by decreasing the resolution bandwidth of 


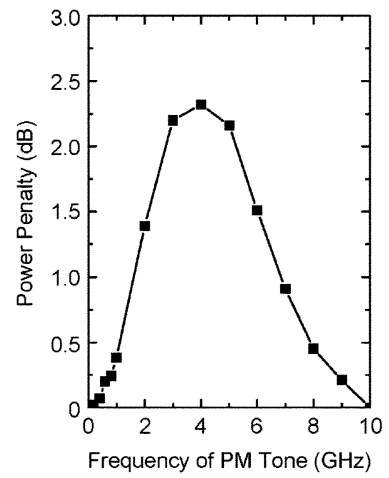

(a)

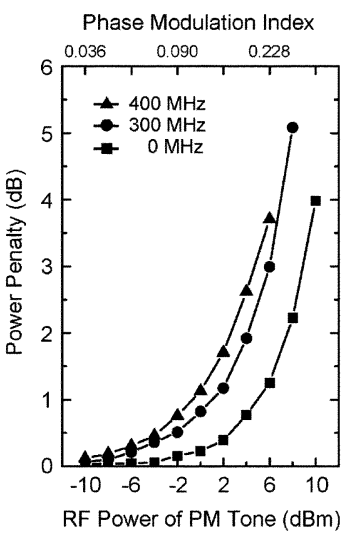

(b)
Fig. 4. Tone-induced sensitivity penalty of the DPSK signals versus (a) PM-tone frequency and (b) PM-tone amplitude.

measurement. The measured results show a similar tendency to the AM fundamental tone [i.e., Fig. 3(b)]: the sensitivity penalty increases with the tone frequency up to half the data rate and then decreases after that. Nevertheless, we choose $4 \mathrm{GHz}$, where the AM fundamental tone is highest in our experiment, for PM-tone frequency since the sensitivity penalty can be reduced by decreasing the tone modulation index.

Fig. 4(b) shows the measured sensitivity penalty of the DPSK signals as a function of the RF power of 4-GHz PM tone. In this measurement, we eliminated the effect of $\mathrm{PD} \lambda$ by minimizing the $\mathrm{PD} \lambda$-induced $\mathrm{AM}$ tone at $4 \mathrm{GHz}$ using a polarization controller (PC) placed at the input of the DI. The measurement results show that the power penalty caused by the PM tone is less than $0.1 \mathrm{~dB}$ as long as the PM-tone power is smaller than $-4 \mathrm{dBm}$. We also depicted in Fig. 4(b) the power penalty versus the PM-tone power in the presence of the frequency offset. In this figure, it should be noted that the power penalties are normalized with respect to the case where no PM-tone is applied to the DPSK signals. Thus, the receiver sensitivities are degraded by 0.4 and $0.5 \mathrm{~dB}$ when the frequency offsets are 300 and $400 \mathrm{MHz}$, respectively. The results clearly show that the power penalty induced by the PM tone increases with the frequency offset. This is because in the presence of the frequency offset the PM tone is more efficiently converted into AM by the DI [5], which is also evident from (3). Based on this measurement, we set the PM-tone power to be $-6 \mathrm{dBm}$ to have the PM-tone-induced power penalty less than $0.3 \mathrm{~dB}$ even when we have a frequency offset of $400 \mathrm{MHz}$.

To evaluate the performance of the proposed monitoring technique, we varied the frequency offset from -2 to $2 \mathrm{GHz}$ with an increment of $100 \mathrm{MHz}$ by changing the temperature of the DFB laser. We then measured the AM-tone power at $4 \mathrm{GHz}$ as well as BER as a function of the frequency offset. In this measurement, we intentionally reduced the OSNR to $\sim 14 \mathrm{~dB}$ to have the BER at around $10^{-9}$. Fig. 5 shows the measured AM-tone power together with the calculated values obtained from (3). The minimum tone power, which occurs in the absence of the frequency offset, was measured to be $-87.7 \mathrm{dBm}$ and the noise power was less than $-100 \mathrm{dBm}$ with a resolution bandwidth of $1 \mathrm{MHz}$. The results show both the BER and the RF power of the AM tone rapidly increase with the frequency offset. In addition, the minimum RF power and the BER are observed when

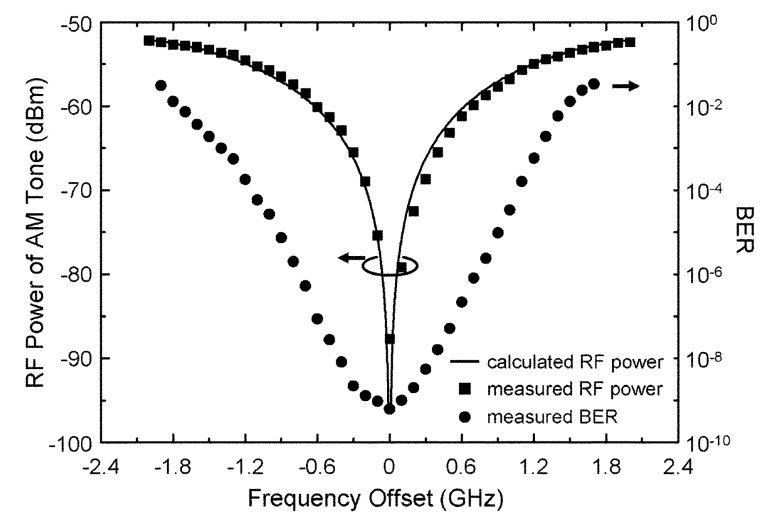

Fig. 5. Measured monitoring signal and BER of the DPSK signal when we intentionally vary the frequency offset. For comparison, the theoretically calculated value is also plotted.

the frequency offset is zero, since we eliminated the effect of $\mathrm{PD} \lambda$ with the PC. The measured data agree well with the calculated values even when the frequency offset is increased up to $\pm 2 \mathrm{GHz}$. This monitoring range of frequency offset would be sufficient for $10-\mathrm{Gb} / \mathrm{s}$ DPSK systems. The monitoring sensitivity is high enough to measure the frequency offset as small as $10 \mathrm{MHz}$, since we observed the RF power variation of AM tone by as large as $\sim 24 \mathrm{~dB}$ for the frequency offset of $\pm 400 \mathrm{MHz}$.

\section{CONCLUSION}

We have proposed and demonstrated a novel technique for monitoring the frequency offset between the laser and DI in direct-detection DPSK systems. To achieve a high monitoring sensitivity, we applied a PM tone to DPSK signals at the transmitter and then detected the RF power of the PM-to-AM-converted tone at the receiver. Our experimental demonstration shows the proposed technique can monitor as large the frequency offset as $\pm 2 \mathrm{GHz}$ with a high sensitivity of $\sim 10 \mathrm{MHz}$. Therefore, the proposed technique can be used for DPSK systems either to generate alarm signals for the frequency offset or to actively control the feedback loop for the DI, thereby enhancing the reliability of the DPSK receiver.

\section{REFERENCES}

[1] H. Kim, "Cross-phase-modulation-induced nonlinear phase noise in WDM direct-detection DPSK systems," J. Lightw. Technol., vol. 21, no. 8, pp. 1770-1774, Aug. 2003.

[2] J. Leibrich, C. Wree, and W. Rosenkranz, "CF-RZ-DPSK for suppression of XPM on dispersion-managed long-haul optical WDM transmission on standard single-mode fiber," IEEE Photon. Technol. Lett., vol. 14, no. 2, pp. 155-157, Feb. 2002.

[3] X. Liu, C. Xu, and X. Wei, "Nonlinear phase noise in pulse-overlapped transmission based on return-to-zero differential phase shift keying," in Proc. ECOC, vol. 4, 2002, Paper 9.6.5.

[4] P. J. Winzer and H. Kim, "Degradations in balanced DPSK receivers," IEEE Photon. Technol. Lett., vol. 15, no. 9, pp. 1282-1284, Sep. 2003.

[5] H. Kim and P. J. Winzer, "Robustness to laser frequency offset in directdetection DPSK and DQPSK systems," J. Lightw. Technol., vol. 21, no. 9, pp. 1887-1891, Sep. 2003.

[6] E. A. Swanson, J. C. Livas, and R. S. Bondurant, "High sensitivity optically preamplified direct detection DPSK receiver with active delay-line stabilization," IEEE Photon. Technol. Lett., vol. 6, no. 2, pp. 263-265, Feb. 1994

[7] S. K. Nielsen, B. F. Skipper, and J. P. Villadsen, "Universal AFC for use in optical DPSK systems," Electron. Lett., vol. 29, pp. 1445-1446, Aug. 1993. 